\title{
Mediation in claims problems
}

\author{
José-Manuel Giménez-Gómez • Josep E. Peris
}

Received: 23 January 2013 / Accepted: 22 April 2014 / Published online: 10 May 2014 (C) The Author(s) 2014. This article is published with open access at SpringerLink.com

\begin{abstract}
Mediation is a dispute resolution process whereby agents reach a mutually acceptable agreement among different proposals that satisfy a set of principles. This paper provides a natural way of coming to such agreements in claims problems. In our approach, mediation combines (i) a set of fair properties (legitimate principles); and (ii) a criterion for delimiting the admissible manners of distributing the endowment, that is determined by the mediator expressing the two (dual) points of view to face such problems: awards and losses. These dual views define a lower and an upper bounds on awards, which are used to implement the so-called Double Recursive Process. We find that this process concludes at the midpoint between the two dual points of view. Finally, we argue that the criterion of the mediator could be established throughout Lorenz domination. In so doing, we retrieve the average of old and well-known rules.
\end{abstract}

Keywords Claims problems $\cdot$ Midpoint $\cdot$ Bounds $\cdot$ Recursivity $\cdot$ Self-duality

JEL Classification $\quad$ C71 $\cdot$ D63 $\cdot$ D71

\section{Introduction}

How should scarce resources be allocated among claimants? Such problems, in which the available quantity of a perfectly divisible good (or endowment) is not sufficient to

J.-M. Giménez-Gómez ( $\varangle)$

Dep. d'Economia and CREIP, Universitat Rovira i Virgili, Av. Universitat 1, 43204 Reus, Spain e-mail: josemanuel.gimenez@urv.cat

\section{J. E. Peris}

Dep. de Mètodes Quantitatius i Teoria Econòmica, Universitat d'Alacant, 03080 Alacant, Spain e-mail: peris@ua.es 
satisfy the aggregate claim against the good, are known as claims problems. The formal analysis of these situations, which originates in a seminal paper by O'Neill (1982), proposes a number of well-behaved rules recommending a division of the resources among agents that depends both on the endowment and on the agents' claims. The term "well-behaved" indicates that these rules may satisfy principles of fairness or other appealing properties. In this context, how can claimants commit to a distribution of resources? How can claimants agree on the properties that must be applied to solve such a problem?

Nowadays, conflicts in which two or more agents cannot decide the allocation have been resolved by a trial. In such cases, a neutral third agent (the mediator) dictates the manner by which the endowment is distributed. This is the philosophy behind mediation, as used in legal, commercial, family and public policy disputes. More precisely, the World Mediation Forum defines mediation "as a process by which a neutral third agent, called a mediator, helps agents in conflict to negotiate a mutually acceptable agreement."

Accordingly, we analyse the figure of a mediator taking as our starting point claims problems with legitimate principles. To this respect, Giménez-Gómez and Marco-Gil (2014) synthesize earlier work to thoroughly analyse the consequences of enriching the classical model of rationing by adding an additional element, $\mathcal{P}$, that represents the legitimate principles set. This set consists of a group of some fair axioms or properties that are commonly accepted by the agents, or that are imposed by law and tradition. Nevertheless, fairness hardly leads to a unique commitment: the same claims problem faced by two different agents may lead to the use of two different points of view (Moulin 1988; Schokkaert and Overlaet 1989; Young 1987, among others). Particularly, two natural dual points of view appear in claims problems: awards and losses. In the former case, each agent is worried about "what she receives", while in the latter one, she cares about "what is not satisfied of her claims".

In the current approach, mediation combines these two dual points of view with the imposition of fair principles. Specifically, a mediator proposes a way of distributing the endowment, $F$, among those that satisfy the legitimate principles $\mathcal{P}$ on which the society has agreed, so that the admissible rules should fulfil $\mathcal{P}$ and propose an allocation between $F$ and its dual rule, $F^{d}$.

Then, we study the consequences of establishing a warranty in awards (lower bound on awards), and the obligation of incurring minimum losses (lower bound on losses, or, equivalently, an upper bound on awards). The idea of establishing a lower bound on awards can be found in many papers on claims problems (O'Neill 1982; Herrero and Villar 2001, 2002; Moulin 2002; Moreno-Ternero and Villar 2004; Giménez-Gómez and Marco-Gil 2014; Dominguez 2013). In fact, the formal definition of a rule already includes both upper and lower bounds on awards by requiring that no agent receives more than her claim or less than zero. Actually, there are many situations where this warranties appear: bankruptcy law, inheritance, divorce, the universal basic income (Tobin et al. 1967; Noguera 2010). On the other hand, since claims problems can be faced from the point of view of the incurred losses, the notion of an upper bound on awards naturally arises. 
From this reasoning, the so-called Double Recursive Process provides a manner to solve claims problems as the recursive application of both: ${ }^{1}$

- a lower bound, which guarantees that each agent should receive at least the smallest amount recommended by all the admissible allocations; and

- an upper bound, which limits the awards an agent should receive by the highest amount provided by these allocations.

Our process can be summarized in the following way: Given a claims problem $(E, c)$, a mediator determines a set of legitimate principles $\mathcal{P}$ and a criterion $\mathcal{D}$ to choose a particular rule $F$ that delimits the set of admissible proposals. Accordingly, the agents compute their minimal expected award (that we call $F$-right) among all admissible proposals; and accept this award. At the same time, they also compute their maximal expected award (that we call $F$-utopia); and give up any claims greater than their utopia. If there are available resources after distributing the rights, the process continues until the endowment is exhausted. We show that this process ends at the midpoint of the two dual proposals, $F$ and $F^{d}$. This result provides a new justification of the average of two extreme and dual ways of distributing the endowment.

The paper is organized as follows: Sect. 2 presents the preliminaries, and Sect. 3 provides our approach. Sections 4 and 5 contain our main definitions and results, respectively. Section 6 applies them to different legitimate principles sets, and Sect. 7 summarizes our final remarks. Finally, the Appendices gather the technical proofs.

\section{Preliminaries}

Given a set of agents $N=\{1,2, \ldots, n\}$, a claims problem is a pair $(E, c) \in \mathbb{R}_{+} \times \mathbb{R}_{+}^{n}$ such that $\sum_{i \in N} c_{i} \geq E$, where $E$ is the endowment, that represents the quantity of a perfectly divisible good to be allocated to the agents, and for each $i \in N, c_{i}$ is the claim that agent $i$ has on the endowment $E$. We will denote by $C=\sum_{i \in N} c_{i}$ the aggregate claim. $\mathfrak{B}$ represents the set of all claims problems.

A rule (claims problems solution) is a function, $\varphi: \mathfrak{B} \rightarrow \mathbb{R}_{+}^{n}$, such that for each $(E, c) \in \mathfrak{B}$, satisfies:

1. $\sum_{i \in N} \varphi_{i}(E, c)=E$ (efficiency); and

2. $0 \leq \varphi_{i}(E, c) \leq c_{i}, \forall i \in N$ (non-negativity and claim-boundedness).

Following Thomson (2013), in claims problems, no claimant should be asked to pay (non-negativity), no claimant should be awarded more than his claim (claimboundedness) and the distributions must be balanced: the sum of the awards should be equal to the endowment (efficiency).

Each claims problem can be faced from two points of view: those of awards and losses. Thus, we have two dual positions depending on whether we are focused on awards or on the unsatisfied demand, $L=\sum_{i \in N} c_{i}-E$. Given a rule $\varphi$, its dual rule distributes "what is missing" in the same way that $\varphi$ divides "what is available" (Aumann and Maschler 1985). Formally,

\footnotetext{
1 The recursive application of a lower bound has been used by Alcalde et al. (2005), Dominguez and Thomson (2006) and Dominguez (2013).
} 
The dual of a rule $\varphi$, denoted by $\varphi^{d}$, is defined by setting for each $(E, c) \in \mathfrak{B}$ and each $i \in N, \varphi_{i}^{d}(E, c)=c_{i}-\varphi_{i}(L, c)$.

It is straightforward to check that the duality operator is well defined, since for each $(E, c) \in \mathfrak{B},(L, c) \in \mathfrak{B}$ and if $\varphi$ satisfies efficiency, non-negativity and claimboundedness, so does $\varphi^{d}$. It is also clear that $\left(\varphi^{d}\right)^{d}=\varphi$.

\subsection{Rules}

In this paper we will focus on some particular rules: the Proportional, the Constrained Equal Awards, and the Constrained Equal Losses rules.

The Proportional $(P)$ rule recommends a division of the endowment proportionally to the claims: for each $(E, c) \in \mathfrak{B}$ and each $i \in N$,

$$
P_{i}(E, c)=\frac{c_{i}}{C} E
$$

The Constrained Equal Awards (CEA) rule (Maimonides, twelfth century, among others) recommends equal awards to all agents, subject to no one receiving more than her claim: for each $(E, c) \in \mathfrak{B}$ and each $i \in N$,

$$
C E A_{i}(E, c) \equiv \min \left\{c_{i}, \mu\right\}
$$

where $\mu$ is chosen so that $\sum_{i \in N} \min \left\{c_{i}, \mu\right\}=E$.

The Constrained Equal Losses rule (CEL), discussed by Maimonides (Aumann and Maschler 1985), is the dual of the Constrained Equal Awards (Herrero and Villar 2001). Specifically, it chooses the awards vector at which all agents incur equal losses, subject to no one receiving a negative amount: for each $(E, c) \in \mathfrak{B}$ and each $i \in N$,

$$
C E L_{i}(E, c) \equiv \max \left\{0, c_{i}-\mu\right\}
$$

where $\mu$ is chosen so that $\sum_{i \in N} \max \left\{0, c_{i}-\mu\right\}=E$.

Note that the dual of the Proportional rule is itself (this property is known as self-duality, see Sect. 2.2).

For the sake of facilitate the understanding of the mentioned rules, we provide the graphical representation (in the two agents case) of these rules, and a simple numerical example.

Example 1 Consider now the claims problem $(E, c)=(15,(8,12))$. Table 1 shows the proposals of the different rules we have introduced.

Table 1 Proposals given by rules, $(E, c)=(15,(8,12))$

\begin{tabular}{lll}
\hline$P$ & $C E A$ & $C E L$ \\
\hline$(6,9)$ & $(7.5,7.5)$ & $(5.5,9.5)$ \\
\hline
\end{tabular}




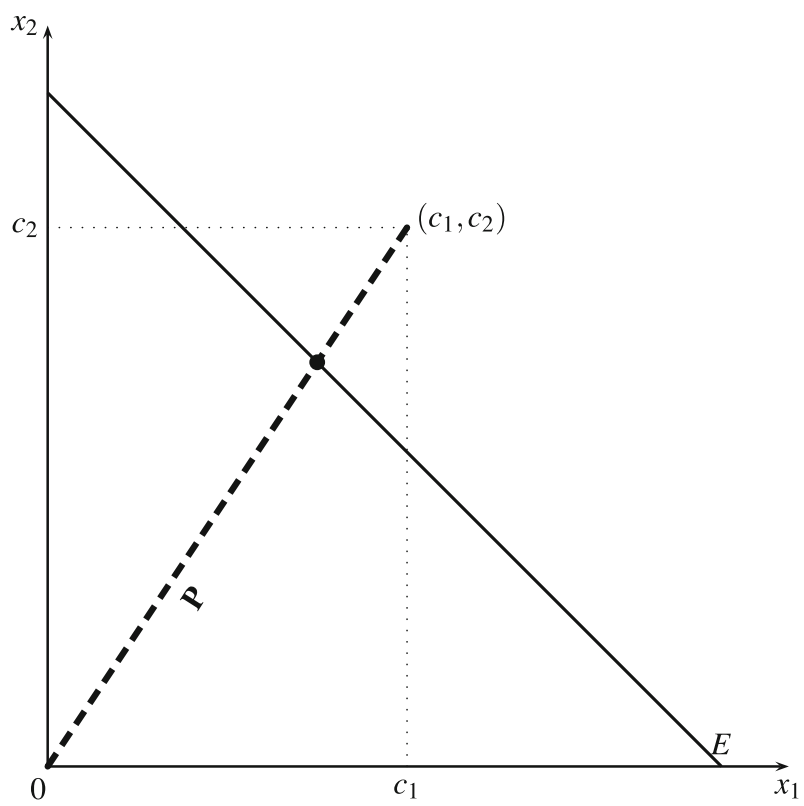

Fig. 1 The Proportional rule

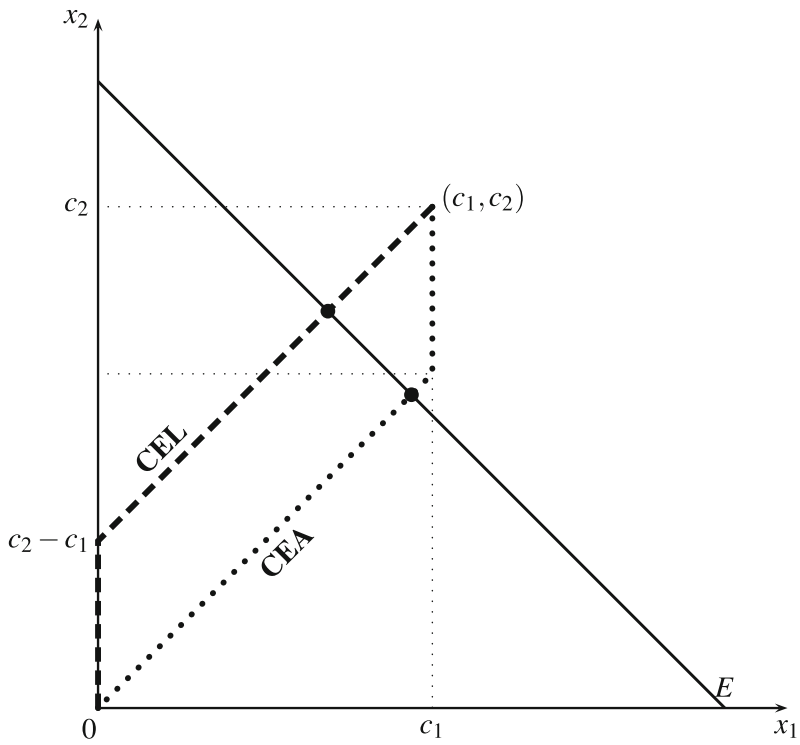

Fig. 2 The CEA and CEL rules

In Figs. 1 and 2 we show the path defined by the above mentioned rules. Then, for a given claims problem $(E, c) \in \mathfrak{B}$ with two agents, the proposed solution is obtained where this path intersects the efficient boundary $x_{1}+x_{2}=E$.

Note that the more properties are required by a society, the fewer the number of admissible rules. 


\subsection{Properties}

We present in this section some properties on the rules that will be useful in our discussion.

Resource monotonicity (Curiel et al. 1987; Young 1987) demands that if the endowment increases, then all individuals should get at least what they received initially. Note that, no rule violating this property has been proposed in claims problems literature. Otherwise we could have situations where an increment of the endowment would cause some disadvantages to certain agents. This property is stated as follows: for each $(E, c) \in \mathfrak{B}$ and each $E^{\prime} \in \mathbb{R}_{+}$such that $C>E^{\prime}>E$, then, for each $i \in N$,

$$
\varphi_{i}\left(E^{\prime}, c\right) \geq \varphi_{i}(E, c) .
$$

Order preservation (Aumann and Maschler 1985) requires that agents with higher claims should receive, and lose, at least as much as agents with lower claims do: for each $(E, c) \in \mathfrak{B}$, and each $i, j \in N$, such that $c_{i} \geq c_{j}$, then

$$
\varphi_{i}(E, c) \geq \varphi_{j}(E, c), \quad \text { and } \quad c_{i}-\varphi_{i}(E, c) \geq c_{j}-\varphi_{j}(E, c) .
$$

Composition up (Moulin 2000) states that if after having distributed the endowment, this increases, then a rule recommends the same if (i) we cancel the initial distribution and apply the rule to the revised problem, or (ii) we let agents keep their initial awards, adjust their claims down by these amounts, and re-apply the rule to allocate the increment of the endowment. For each $(E, c) \in \mathfrak{B}$, each $E^{\prime} \in \mathbb{R}_{+}$such that $C>E^{\prime}>E$, then

$$
\varphi\left(E^{\prime}, c\right)=\varphi(E, c)+\varphi\left(E^{\prime}-E, c-\varphi(E, c)\right) .
$$

Self-duality (Aumann and Maschler 1985) demands that a rule recommends the same allocation for a claims problem $(E, c)$ when dividing "what is available" (awards) or "what is missing" (losses). That is, the rule coincides with its dual: for each $(E, c) \in$ $\mathfrak{B}$,

$$
\varphi(E, c)=\varphi^{d}(E, c)
$$

Table 2 summarizes the properties that fulfil the solutions we work on.

Table 2 Properties and rules

\begin{tabular}{llll}
\hline & $C E A$ & $C E L$ & $P$ \\
\hline Resource monotonicity & Yes & Yes & Yes \\
Order preservation & Yes & Yes & Yes \\
Composition up & Yes & Yes & Yes \\
Self-duality & No & No & Yes \\
\hline
\end{tabular}




\section{Mediation}

Our notion of mediation is given by both a set of properties and an admissible zone that selects some of the rules that satisfy those properties. First, a group of properties are accepted by a particular society (either these properties can be assumed by the agents involved in the problem, or they can be established by law). ${ }^{2}$ Then, among the rules fulfilling these properties, a reference rule $F$ and its dual rule $F^{d}$ delimit the set of proposals that are admissible by a particular mediator. In this sense, mediation involves:

1. a set of legitimate principles (properties) $\mathcal{P}$;

2. a criterion $\mathcal{D}$ that selects a reference rule $F$, satisfying the properties in $\mathcal{P}$; and

3. an admissible zone delimited by rule $F$ and its dual $F^{d}$.

In Sect. 6 we propose the Lorenz domination as the criterion to be used in order to select the reference rule $F$. So, we consider first a society in which the distribution of the endowment must be based on the selected reference rule, that delimits a set of admissible allocations for a specific mediator. Next, we formally present our model.

Definition 1 A claims problem with mediation is a triplet $(E, c, F)$, wherein $(E, c) \in \mathfrak{B}$, and $F$ is a fixed rule determined from a set of principles $\mathcal{P}$ upon which a particular society has agreed. Let $\mathfrak{B}_{F}$ be the set of all claims problems with mediation.

Then, we identify the mediator with the rule $F$, that will delimit the admissible zone. Although $F$ depends on the set $\mathcal{P}$ of legitimate principles, and the criterion $\mathcal{D}$ used, $F=F(\mathcal{P}, \mathcal{D})$, we omit this fact in the notation, unless needed. We now formally define the admissible rules for a given mediator.

Definition 2 A rule $\varphi: \mathfrak{B} \rightarrow \mathbb{R}_{+}^{n}$ is said to be admissible for mediator $F=F(\mathcal{P}, \mathcal{D})$ if $\varphi$ satisfies all properties in $\mathcal{P}$ and for all $i \in N$,

$$
\min \left\{F_{i}(E, c), F_{i}^{d}(E, c)\right\} \leq \varphi_{i}(E, c) \leq \max \left\{F_{i}(E, c), F_{i}^{d}(E, c)\right\} .
$$

Let $\Phi(F)$ denote the set of admissible rules for mediator $F$.

Example 2 (Proportional mediator) Consider a mediator such that she asks the rules to fulfill self-duality and composition-up. In this case, the only rule that satisfies these properties is the Proportional. So that, $F=P$. Since this solution is self-dual, $F=$ $F^{d}$, the only admissible rule is the Proportional one. In this case, the criterion of the mediator determines directly the solution.

Example 3 (Egalitarian mediator) Consider a mediator such that every rule is accepted and assume that the criterion to select one of the rules (see Sect. 6) determines $F=C E A$. Then, the admissible rules are those whose proposals for each agent $i$ are between the values $C E A_{i}(E, c)$ and $C E L_{i}(E, c)$.

\footnotetext{
${ }^{2}$ For instance, "any bankruptcy rule that is in line with the existing laws as described in the USA Bankruptcy Code is necessarily population monotonic" (Grahn and Voorneveld 2002).
} 
For instance, if $(E, c)=(15,(8,12))$, the admissible rules consist of the proposals within the set $\left\{(a, b) \in \mathbb{R}^{2}: a+b=15: 5.5 \leq a \leq 7.5\right\}$, see Fig. 2 . In this case the distribution of the endowment is undetermined and a process is needed to obtain a solution for the claims problem.

\section{The double recursive process}

Given the set of admissible rules for mediator $F, \Phi(F)$, we can obtain a minimal and a maximal level of awards that each agent should receive on the basis of the ordinary meaning of a guarantee. The maximal level on awards implies a limit on the losses the agent incurs. In this sense, we can associate to every claims problem with mediation two bounds on the awards each agent should receive:

- the $F$-rights value, which is a lower bound on awards that provides each agent the smallest amount recommended to her by all admissible rules (see Giménez-Gómez and Marco-Gil 2014); and

- the F-utopia value, which is an upper bound that limits the awards that each agent can receive to the highest amount recommended to her by all admissible rules.

Definition 3 Given $(E, c, F) \in \mathfrak{B}_{F}$, the $\boldsymbol{F}$-rights value, $r$, is for each $i \in N$,

$$
r_{i}(E, c, F)=\min _{\varphi \in \Phi(F)}\left\{\varphi_{i}(E, c)\right\}
$$

Definition 4 Given $(E, c, F) \in \mathfrak{B}_{F}$, the $\boldsymbol{F}$-utopia value, $u$, is for each $i \in N$,

$$
u_{i}(E, c, F)=\max _{\varphi \in \Phi(F)}\left\{\varphi_{i}(E, c)\right\}
$$

Remark 1 Given a particular claims problem $(E, c)$ the admissible rule that provides the $F$-rights ( $F$-utopia) for agent $i$, could be different from the admissible rule that provides the $F$-rights ( $F$-utopia) for agent $j \neq i$.

The F-rights can be understood as the part of the claims that are not disputed (an idea that has been already used in claims problems (see, for instance, Aumann and Maschler 1985)), so that it should be ensured to each agent. Furthermore, assigning to each agent her F-rights (as we have defined it) is compatible with the existing endowment $E$, that is $\sum_{i \in N} r_{i} \leq E$. Moreover, all the admissible rules recommend to each agent at least this amount. As, in general, the sum of the agents' $F$-rights does not exhaust the endowment, a recursive process arises in a natural way in order to obtain an efficient allocation: we can allocate her $F$-rights to any agent, and then proceed again in the new problem defined by the remaining estate ( $E$ minus the allocated amounts), and the claims resulting from subtracting to the initial ones the amount which has already been granted $\left(c_{i}-r_{i}\right)$. As we will see (Example 5) this process may end up at one of the extremes of the admissible zone, defined by the reference rule $F$ and its dual.

By applying the idea of guaranteeing a minimum amount on losses rather than awards, we propose truncate the claims by the maximum amount that each agent can 
expect to receive, the $F$-utopia. The idea of truncate the claims is not new and, in fact, it is also commonly assumed that the part of the claims that are not feasible should not to be considered in the problem (see, for instance, Aumann and Maschler 1985).

Therefore, we define an iterative process, that we call the Double Recursive Process in which, at any step, each agent receives her F-rights and, at the same time, her claim is truncated by her F-utopia.

Definition 5 The Double Recursive Process, $D R P$, associates for each $(E, c, F) \in$ $\mathfrak{B}_{F}$ and each $i \in N$,

$$
D R P_{i}(E, c)=\sum_{m=1}^{\infty} r_{i}\left(E^{m}, c^{m}, F\right),
$$

where for each step $m \in \mathbb{N}, r_{i}\left(E^{m}, c^{m}, F\right)$ is defined in the following way:

step 1 , for $m=1$, define $\left(E^{1}, c^{1}\right)=(E, c)$ and compute

$$
\begin{aligned}
& r_{i}\left(E^{1}, c^{1}, F\right)=\min _{\varphi \in \Phi(F)}\left\{\varphi_{i}\left(E^{1}, c^{1}\right)\right\}, \\
& u_{i}\left(E^{1}, c^{1}, F\right)=\max _{\varphi \in \Phi(F)}\left\{\varphi_{i}\left(E^{1}, c^{1}\right)\right\} .
\end{aligned}
$$

step $m$, for $m \geq 2$, define

$$
\begin{aligned}
E^{m} & =E^{m-1}-\sum_{i \in N} r_{i}\left(E^{m-1}, c^{m-1}, F\right), \\
c_{i}^{m} & =u_{i}\left(E^{m-1}, c^{m-1}, F\right)-r_{i}\left(E^{m-1}, c^{m-1}, F\right),
\end{aligned}
$$

and compute

$$
\begin{aligned}
& r_{i}\left(E^{m}, c^{m}, F\right)=\min _{\varphi \in \Phi(F)}\left\{\varphi_{i}\left(E^{m}, c^{m}\right)\right\}, \\
& u_{i}\left(E^{m}, c^{m}, F\right)=\max _{\varphi \in \Phi(F)}\left\{\varphi_{i}\left(E^{m}, c^{m}\right)\right\} .
\end{aligned}
$$

As shown in Theorem 1 this process completely exhausts the endowment $E$, so it provides an efficient allocation. In Example 4 we show this process in a particular claims problem with mediation.

Example 4 Consider the Egalitarian mediator as in Example 3, and the claims problem $(E, c)=(15,(8,12))$. We know that the reference rule is $F=C E A$, so $F^{d}=C E L$. Then,

$$
\begin{aligned}
& m=1: E^{1}=15 ; c^{1}=(8,12) ; \\
& C E A\left(E^{1}, c^{1}\right)=(7.5,7.5) ; \quad C E L\left(E^{1}, c^{1}\right)=(5.5,9.5) ; \\
& r\left(E^{1}, c^{1}, F\right)=(5.5,7.5) ; \quad u\left(E^{1}, c^{1}, F\right)=(7.5,9.5) .
\end{aligned}
$$




$$
\begin{gathered}
m=2: E^{2}=2 ; c^{2}=(2,2) ; \\
C E A\left(E^{2}, c^{2}\right)=(1,1)=C E L\left(E^{2}, c^{2}\right) ; \\
r\left(E^{2}, c^{2}, F\right)=(1,1)=u\left(E^{2}, c^{2}, F\right) . \\
m=3: E^{3}=0 ; \quad c^{3}=(0,0) .
\end{gathered}
$$

Therefore, $\operatorname{DRP}(E, c)=(5.5,7.5)+(1,1)=(6.5,8.5)$.

\section{Main results}

Our first result proves that the Double Recursive Process ends at the midpoint of the allocations provided by $F$ and $F^{d}$.

Theorem 1 For each $(E, c, F) \in \mathfrak{B}_{F}$

$$
\operatorname{DRP}(E, c)=\frac{F(E, c)+F^{d}(E, c)}{2}
$$

Proof See Appendix A.

The next theorem establishes that the final allocation provided by the Double Recursive Process corresponds with the average of the F-rights and the F-utopia. The proof is obtained as part of the proof of Theorem 1.

Theorem 2 For each $(E, c, F) \in \mathfrak{B}_{F}$

$$
D R P(E, c)=\frac{r(E, c, F)+u(E, c, F)}{2}
$$

Theorem 1 shows that the Double Recursive Process proposes the midpoint between the two rules which represent extreme and opposite ways of sharing awards among claimants. As a consequence, it can be stated that the rationing of the endowment obtained by the recursive double imposition of the F-rights and F-utopia, neither favours nor hurts any particular agent. Following Thomson and Yeh (2008),

"When two rules express opposite points of view on how to solve a bankruptcy problem, it is natural to compromise between them by averaging”.

Note that, from Theorem 1, we obtain that the Double Recursive Process satisfies efficiency, non-negativity and claim-boundedness. Furthermore, the following result shows that it also fulfils self-duality, whatever the legitimate principles set, $\mathcal{P}$, and the reference rule, $F$, are.

Proposition 1 For each $(E, c, F) \in \mathfrak{B}_{F}, D R P(E, c)=c-D R P(L, c)$.

Proof See Appendix B. 
In our context of claims problems with mediation, we understand by a process any algorithm that associates to each problem $(E, c, F) \in \mathfrak{B}_{F}$ an allocation satisfying efficiency, non-negativity and claim-boundedness. For instance, apart from our Double Recursive Process, we may define a process that in each step assigns the $F$-rights value, and solves the residual problem, as shown by next example.

Example 5 Consider the Egalitarian mediator as in Example 3, and the claims problem $(E, c)=(15,(8,12))$. We know that the reference rule is $F=C E A$, so $F^{d}=C E L$. Then, we define a recursive process $M_{r}$ in the following way:

$$
\left(M_{r}\right)_{i}=\sum_{m=1}^{\infty} r_{i}\left(E^{m}, c^{m}, F\right)
$$

where $E^{m}$ is as in Definition 5, and $c^{m}=c^{m-1}-r^{m-1}$ for all $m$. Then

$$
\begin{gathered}
m=1: E^{1}=15 ; c^{1}=(8,12) \\
C E A\left(E^{1}, c^{1}\right)=(7.5,7.5) ; \quad C E L\left(E^{1}, c^{1}\right)=(5.5,9.5) ; \\
r^{1}=r\left(E^{1}, c^{1}, F\right)=(5.5,7.5)
\end{gathered}
$$$$
C E A\left(E^{2}, c^{2}\right)=(1,1) ; \quad C E L\left(E^{2}, c^{2}\right)=(0,2) ;
$$$$
r^{2}=r\left(E^{2}, c^{2}, F\right)=(0,1)
$$$$
m=3: E^{3}=1 ; c^{3}=(2.5,3.5)
$$

$$
\begin{aligned}
C E A\left(E^{3}, c^{3}\right) & =(0.5,0.5) ; \quad C E L\left(E^{3}, c^{3}\right)=(0,1) ; \\
r^{3} & =r\left(E^{3}, c^{3}, F\right)=(0,0.5)
\end{aligned}
$$

$m=k:$

$$
r^{k}=r\left(E^{k}, c^{k}, F\right)=\left(0,(1 / 2)^{k-2}\right)
$$

Therefore, $M_{r}(E, c)=(5.5,9.5)$, that does not coincide with the allocation provided by the Double Recursive Process, $D R P(E, c)=(6.5,8.5)$.

Now, let us consider the "extra awards" each agent receives from a process, i.e., the amount of awards each agent gets apart from what she has guaranteed, $r_{i}(E, c)$. Given that the process we are looking for is self dual, we ask that the extra amount each individual receives after rights should be the same in a problem $(E, c)$ and in its dual problem $(L, c)$. We call this property dual right-egalitarian:

$$
M_{i}(E, c)-r_{i}(E, c)=M_{i}(L, c)-r_{i}(L, c) .
$$

Now, by combining self-duality and dual right-egalitarian, we obtain a characterization of the Double Recursive Process. 
Theorem 3 If $M$ is a process such that for each $(E, c, F) \in \mathfrak{B}_{F}$, satisfies self-duality and dual right-egalitarian, then $M(E, c)=D R P(E, c)$.

Proof See Appendix C.

Finally, note that there are some properties that are always satisfied by the result of this process independently of the legitimate principles set, $\mathcal{P}$, and the reference rule, $F$. Among the properties we have introduced in Sect. 2.2, the Double Recursive Process fulfils: resource monotonicity, order preservation and self-duality. Nonetheless, other properties depend on $\mathcal{P}$ and/or on $F$, as the next example shows with respect to the composition-up property.

Example 6 Consider $F=C E A$ (the Egalitarian mediator) and the problems $(E, c)=$ $(100,(80,120))$ and $\left(E^{\prime}, c\right)=(120,(80,120))$. Then,

$$
\begin{aligned}
& D R P(E, c)=(40,60), \quad D R P\left(E^{\prime}, c\right)=(50,70) \\
& \text { and } D R P(20,(40,60))=(5,15) .
\end{aligned}
$$

So composition- $u p$ is not fulfilled. Now, consider $F=P$ (the Proportional mediator). In this case, $D R P$ provides an allocation that always coincides with the proportional one, so it satisfies composition-up.

\section{The mediator: reference rules}

In Sect. 3 we have introduced the notion of mediation as a specific rule $F$, that fulfills a set of legitimate principles, $\mathcal{P}$, and determines the set of admissible rules. Now, we provide some rationale about the selection of the reference rule, $F$, by the mediator. As we have mentioned in Sect. 1, the Lorenz criterion is a usual equity manner of comparing rules (see Thomson 2012; Bosmans and Lauwers 2011). Under some usual conditions, this criterion provides a comparison of rules so that if a rule $\varphi$ Lorenz dominates a rule $\psi$, this means that $\varphi$ favours smaller claimants relative to larger ones. Under such a perspective, the Lorenz maximal rule can be considered as the more equitable one. Then, once the legitimate principles have been defined by society, the Lorenz-optimality can be the criterion the mediator uses to select the reference rule $F$. We call this Lorenz mediation, which involves: (1) a set of properties, $\mathcal{P}$ (legitimate principles); and (2) an admissible zone delimited by the Lorenz maximal rule in $\mathcal{P}, F=\mathcal{L}$, and its dual rule, $F^{d}=\mathcal{L}^{d}$. Therefore, as a consequence of Theorem 1 we obtain that the Double Recursive Process coincides with the average of the Lorenz maximal and its dual.

Then, by varying the set of legitimate principles, the reference rule may change and so the result of the $D R P$ process. The following example and results show this fact. ${ }^{3}$

Example 7 Let us consider the following sets of legitimate principles, and the Lorenzmaximal in each one of them:

\footnotetext{
3 The results about Lorenz maximal rules provided in this example can be found in Bosmans and Lauwers (2011) and Thomson (2012).
} 
- If $\mathcal{P}_{1}=$ \{efficiency, claim-boundedness, non-negativity $\}, \mathcal{L}=C E A$.

- If $\mathcal{P}_{2}=$ \{efficiency, claim-boundedness, non-negativity, composition-up, selfduality\}, $\mathcal{L}=P$.

Proposition 2 Given a society with legitimate principles $\mathcal{P}$, such that a Lorenz maximal $\mathcal{L}$ exists, if $F=\mathcal{L}$, then for each $(E, c, F) \in \mathfrak{B}_{F}$,

$$
D R P(E, c)=\frac{\mathcal{L}(E, c)+\mathcal{L}^{d}(E, c)}{2} .
$$

By applying the result in Proposition 2 to the different sets of legitimate principles introduced in previous example, we obtain the following:

Corollary 1 We consider the sets of legitimate principles defined in Example 7. Then,

(a) If $\mathcal{P}=\mathcal{P}_{1}, \quad D R P(E, c)=\frac{C E A(E, c)+C E L(E, c)}{2}$

(b) If $\mathcal{P}=\mathcal{P}_{2}, \quad D R P(E, c)=P(E, c)$

\section{Final remarks}

In this paper, we have established that the application of a recursive process, in contexts where two dual reference criterion $F$ and $F^{d}$ appear, retrieves the midpoint between these two dual positions. This fact, apart from its own logic, allows us to anticipate the result of such negotiations. Moreover, we also find that this process concludes at the midpoint between the F-rights and the F-utopia - that is, the midpoint between the worst and the best position, among the proposals given by the admissible rules.

Two interesting questions remain open. On the one hand, what happens if instead of considering an admissible zone delimited by a reference function, $F$, and its dual, $F^{d}$, we consider that this zone can be delimited by two any reference functions, $F$ and $G$, satisfying $\mathcal{P}$. In this case the admissible rules are those satisfying $\mathcal{P}$ such that:

$$
\min \left\{F_{i}(E, c), G_{i}(E, c)\right\} \leq \varphi_{i}(E, c) \leq \max \left\{F_{i}(E, c), G_{i}(E, c)\right\}
$$

The Double Recursive Process may be adapted to this situation merely by changing $F^{d}$ by $G$ in Definition 5 . We denote by $D R P 2$ this new process. Next proposition tells us that the result in Theorem 1 remains true if we consider two-agent problems. Nevertheless, we cannot generalize this result to $n$-agent problems, as shown in Example 8.

Proposition 3 For each two-agent claims problems $(E, c) \in \mathfrak{B}$, and given two any reference rules, $F$ and $G$,

$$
D R P 2(E, c)=\frac{F(E, c)+G(E, c)}{2}
$$

Proof See Appendix D. 
Example 8 Consider that the admissible zone is delimited by the Constrained Equal Awards, $C E A$, and Proportional, $P$, rules. Let us consider the claims problem $(E, c)=$ $(100,(50,70,80))$. Then, we obtain that

$$
D R P 2=\left(28+\frac{3}{4}, 34+\frac{7}{12}, 36+\frac{2}{3}\right),
$$

which does not coincide with the average of $C E A(E, c)=\left(\frac{100}{3}, \frac{100}{3}, \frac{100}{3}\right)$, and $P(E, c)=(25,35,40)$.

On the other hand, as suggested by a referee "sometimes, the lower bound $r(E, c, F)$ may be considered as too low (or even to high), we would like to impose an adjusted $F$-right $r^{\alpha}(E, c, P)=\alpha \cdot r(E, c, F)$." So, what happens if we consider some speed in the recursive process by means of a parameter $\alpha$ ? This is an interesting question since it seems that some relationship between the end of the recursive process and some convex combination of the rules $F$ and $F^{d}$ should exist. Nevertheless, no general relationship is fulfilled.

Finally, in Sect. 6 we have provided a way of selecting the reference rule $F$, that coincides with the Lorenz maximal rule in a specific set of legitimate principles. However, there are other possibilities for comparing rules and, so that, defining such a rule (see, for instance, the Rawls criterion Rawls 1971). In any case, the Double Recursive Process ends at the midpoint of any two dual reference rules, $F$ and $F^{d}$.

Acknowledgments We are particularly grateful to two anonymous referees and the Editor for many valuable comments and suggestions that have led to a substantial improvement in the manuscript. The usual caveat applies. Financial support from Universitat Rovira i Virgili, Banco Santander and Generalitat de Catalunya under project 2011LINE-06 are gratefully acknowledged.

Open Access This article is distributed under the terms of the Creative Commons Attribution License which permits any use, distribution, and reproduction in any medium, provided the original author(s) and the source are credited.

\section{Appendix A: Proof of Theorem 1}

The proof of this result is based on a fact and two lemmas. Throughout the appendix, we will consider $(E, c, F) \in \mathfrak{B}_{F}$. For notational convenience, for all $m \in \mathbb{N}$, we denote $r^{m}=r\left(E^{m}, c^{m}, F\right)$ and $u^{m}=u\left(E^{m}, c^{m}, F\right)$.

Fact 1 For all $m \in \mathbb{N}$, the sum of $F$-rights plus $F$-utopia values equals two times the endowment,

$$
\sum_{i \in N} r_{i}^{m}+\sum_{i \in N} u_{i}^{m}=2 E^{m}
$$

Lemma 1 For all $m \in \mathbb{N}, m>1$,

$$
\sum_{i \in N}\left[u_{i}^{m}+r_{i}^{m}\right]=\sum_{i \in N} c_{i}^{m}=C^{m} .
$$


Proof From Fact 1, the next expression follows

$$
\sum_{i \in N}\left[\frac{u_{i}^{m}+r_{i}^{m}}{2}\right]=E^{m} .
$$

By the definition of the Double Recursive Process, we know that

$$
\begin{aligned}
E^{m} & =E^{m-1}-\sum_{i \in N} r_{i}^{m-1} \\
& =\sum_{i \in N}\left[\frac{u_{i}^{m-1}+r_{i}^{m-1}}{2}\right]-\sum_{i \in N} r_{i}^{m-1} \\
& =\sum_{i \in N}\left[\frac{u_{i}^{m-1}-r_{i}^{m-1}}{2}\right]=\frac{\sum_{i \in N} c_{i}^{m}}{2}=\frac{C^{m}}{2} .
\end{aligned}
$$

As a direct consequence of Lemma 1, we find that at every step of the Double Recursive Process, $m \in \mathbb{N}, m>1$, the half of the claims sum coincides with both the endowment and the total loss incurred at this step of the process,

$$
\sum_{i \in N} c_{i}^{m}-E^{m}=E^{m}=\frac{C^{m}}{2}
$$

Finally, the next lemma states that each agent's claim at every step different from the initial one, coincides with sum of the rights and the utopia.

Lemma 2 For all $m \in \mathbb{N}, m>1$

$$
c_{i}^{m}=u_{i}^{m}+r_{i}^{m}
$$

Proof We know that

$$
\sum_{i \in N} c_{i}^{m}-E^{m}=E^{m}
$$

so

$$
\begin{aligned}
r_{i}^{m} & =r_{i}\left(\sum_{i \in N} c_{i}^{m}-E^{m}, c^{m}, F\right)=\min _{\varphi \in \Phi(F)}\left\{\varphi_{i}\left(\sum_{i \in N} c_{i}^{m}-E^{m}, c^{m}, F\right)\right\} \\
& =\min _{\varphi \in \Phi(F)}\left\{c_{i}^{m}-\varphi_{i}^{d}\left(\sum_{i \in N} c_{i}^{m}-E^{m}, c^{m}, F\right)\right\} \\
& =c_{i}^{m}-\max _{\varphi \in \Phi(F)}\left\{\varphi_{i}^{d}\left(\sum_{i \in N} c_{i}^{m}-E^{m}, c^{m}, F\right)\right\}
\end{aligned}
$$




$$
=c_{i}^{m}-u_{i}^{m}\left(\sum_{i \in N} c_{i}^{m}-E^{m}, c^{m}, F\right)=c_{i}^{m}-u_{i}^{m},
$$

and the result holds.

\section{A.1 Proof of Theorem 1}

Proof By the definition of the Double Recursive Process,

$$
\begin{aligned}
\sum_{m=2}^{\infty} c_{i}^{m} & =\sum_{m=2}^{\infty}\left[u_{i}^{m-1}-r_{i}^{m-1}\right] \\
& =u_{i}^{1}+\sum_{m=2}^{\infty} u_{i}^{m}-r_{i}^{1}-\sum_{m=2}^{\infty} r_{i}^{m}
\end{aligned}
$$

By Lemma 2,

$$
\sum_{m=2}^{\infty} c_{i}^{m}=\sum_{m=2}^{\infty}\left[u_{i}^{m}+r_{i}^{m}\right]
$$

So,

$$
u_{i}^{1}+\sum_{m=2}^{\infty} u_{i}^{m}-r_{i}^{1}-\sum_{m=2}^{\infty} r_{i}^{m}=\sum_{m=2}^{\infty}\left[u_{i}^{m}+r_{i}^{m}\right]
$$

Thus,

$$
\sum_{m=2}^{\infty} r_{i}^{m}=\frac{u_{i}^{1}-r_{i}^{1}}{2}
$$

Therefore,

$$
D R P_{i}(E, c)=r_{i}^{1}+\frac{u_{i}^{1}-r_{i}^{1}}{2}=\frac{u_{i}(E, c, F)+r_{i}(E, c, F)}{2}
$$

Now, from definition of $F$-rights and $F$-utopia values, $u_{i}(E, c, F)+r_{i}(E, c, F)=$ $\max \left\{F_{i}(E, c), F_{i}^{d}(E, c)\right\}+\min \left\{F_{i}(E, c), F_{i}^{d}(E, c)\right\}=F_{i}(E, c)+F_{i}^{d}(E, c)$ and the required result holds. 


\section{Appendix B: Proof of Proposition 1}

Let $(E, c, F) \in \mathfrak{B}_{F}$ a claims problem with mediation. By duality,

$$
F(L, c)=c-F^{d}(E, c) \text { and } F^{d}(L, c)=c-F(E, c) .
$$

Since

$$
D R P(E, c)=\frac{F(E, c)+F^{d}(E, c)}{2},
$$

then

$$
\begin{aligned}
D R P^{d}(E, c) & =c-\frac{F(L, c)+F^{d}(L, c)}{2}=c-\frac{c-F^{d}(E, c)+c-F(E, c)}{2} \\
& =\frac{F(E, c)+F^{d}(E, c)}{2}=\operatorname{DRP}(E, c) .
\end{aligned}
$$

\section{Appendix C: Proof of Theorem 3}

Let $(E, c, F) \in \mathfrak{B}_{F}$ a claims problem with mediation. If $M$ is a process that fulfils self-duality, we know that

$$
M_{i}(E, c)=M_{i}^{d}(E, c)=c_{i}-M_{i}(L, c) .
$$

On the other hand, by applying the dual right-egalitarian property

$$
M_{i}(E, c)-r_{i}(E, c)=M_{i}(L, c)-r_{i}(L, c) .
$$

By adding both equalities, we obtain

$$
\begin{aligned}
2 M_{i}(E, c)=c_{i}+r_{i}(E, c)-r_{i}(L, c) & =c_{i}+r_{i}(E, c)-c_{i}+u_{i}(E, c) \\
& =r_{i}(E, c)+u_{i}(E, c),
\end{aligned}
$$

so,

$$
M(E, c)=\frac{r_{i}(E, c)+u_{i}(E, c)}{2}=\operatorname{DRP}(E, c) .
$$




\section{Appendix D: Proof of Proposition 3}

Let $(E, c) \in \mathfrak{B}$ be a two-agent claims problem, and consider that there exist two reference functions $F$ and $G$ that delimit the admissible zone of proposals. That is, a rule $\varphi$ is admissible if satisfies the properties in $\mathcal{P}$ and

$$
\min \left\{F_{i}(E, c), G_{i}(E, c)\right\} \leq \varphi_{i}(E, c) \leq \max \left\{F_{i}(E, c), G_{i}(E, c)\right\}
$$

Suppose, without loss of generality that $c_{1} \leq c_{2}$ and that $F_{1}(E, c)<G_{1}(E, c)$. For notational convenience, for all $m \in \mathbb{N}$, we write $r^{m}=\operatorname{pr}\left(E^{m}, c^{m}, F, G\right), u^{m}=$ $u\left(E^{m}, c^{m}, F, G\right), F^{m}=F\left(E^{m}, c^{m}\right)$, and $G^{m}=G\left(E^{m}, c^{m}\right)$. By the definition of the Double Recursive Process,

- $m=1, E^{1}=E ; c^{1}=c$.

Since $F_{1}^{1}<G_{1}^{1}, \quad r^{1}=\left(F_{1}^{1}, G_{2}^{1}\right) ; \quad u^{1}=\left(G_{1}^{1}, F_{2}^{1}\right)$.

- $m=2, E^{2}=E-F_{1}^{1}-G_{2}^{1} ; c^{2}=\left(G_{1}^{1}-F_{1}^{1}, F_{2}^{1}-G_{2}^{1}\right)$.

Note that, $F_{1}^{1}+F_{2}^{1}=G_{1}^{1}+G_{2}^{1}$. So,

$$
G_{1}^{1}-F_{1}^{1}=F_{2}^{1}-G_{2}^{1} \text {, and } c_{1}^{2}=c_{2}^{2} \text {. }
$$

Hence,

$$
F_{1}^{2}=F_{1}^{2}=G_{1}^{2}=G_{1}^{2}=\frac{E-F_{1}^{1}-G_{1}^{2}}{2} .
$$

Therefore, since $E=G_{1}^{1}+G_{1}^{2}$,

$$
D R P 2_{1}(E, c)=F_{1}^{1}+\frac{E-F_{1}^{1}-G_{1}^{2}}{2}=F_{1}^{1}+\frac{G_{1}^{1}-F_{1}^{1}}{2}=\frac{F_{1}+G_{1}}{2} \text {, }
$$

and, by efficiency,

$$
D R P 2_{2}(E, c)=E-\frac{F_{1}+G_{1}}{2}=\frac{F_{2}+G_{2}}{2} .
$$

\section{References}

Alcalde J, Marco-Gil MC, Silva JA (2005) Bankruptcy games and the Ibn Ezras proposal. Econ Theory 26:103-114

Aumann RJ, Maschler M (1985) Game theoretic analysis of a bankruptcy from the Talmud. J Econ Theory 36:195-213

Bosmans K, Lauwers L (2011) Lorenz comparisons of nine rules for the adjudication of conflicting claims. Int J Game Theory 40:791-807

Curiel J, Maschler M, Tijs S (1987) Bankruptcy games. Z Oper Res 31:143-159

Dominguez D (2013) Lower bounds and recursive methods for the problem of adjudicating conflicting claims. Soc Choice Welf 40:663-678

Dominguez D, Thomson W (2006) A new solution to the problem of adjudicating conflicting claims. Econ Theory 28:283-307. doi:10.1007/s00199-005-0636-3 
Giménez-Gómez JM, Marco-Gil MC (2014) A new approach for bounding awards in bankruptcy problems. SCW. doi:10.1007/s00355-013-0789-0

Grahn S, Voorneveld M (2002) Population monotonic allocation schemes in bankruptcy games. Ann Oper Res 109:317-329

Herrero C, Villar A (2001) The three musketeers: four classical solutions to bankruptcy problems. Math Soc Sci 42:307-328. doi:10.1016/S0165-4896(01)00075-0

Herrero C, Villar A (2002) Sustainability in bankruptcy problems. Top 10:261-273. doi:10.1007/ BF02579019

Moreno-Ternero JD, Villar A (2004) The Talmud rule and the securement of agents' awards. Math Soc Sci 47:245-257. doi:10.1016/S0165-4896(03)00087-8

Moulin H (1988) Axiomatic of cooperative decisions making. Cambridge University Press, Cambridge

Moulin H (2000) Priority rules and other asymmetric rationing methods. Econometrica 68:643-684

Moulin H (2002) Axiomatic cost and surplus sharing. In: Arrow AK, Sen K (eds) Handbook of social choice and welfare, vol 1. Elsevier/North Holland, Amsterdam, pp 289-357

Noguera JA (2010) The universal basic income: reasons and strategies. Policy Papers 5

O’Neill B (1982) A problem of rights arbitration from the Talmud. Math Soc Sci 2:345-371

Rawls J (1971) A theory of justice. Belknap/Harvard, Cambridge

Schokkaert E, Overlaet B (1989) Moral Intuitions and economic models of distributive justice. Soc Choice Welf 6:19-31

Thomson W (2012) Lorenz rankings of rules for the adjudication of conflicting claims. Econ Theory 50:547-569

Thomson W (2013) Axiomatic and game-theoretic analysis of bankruptcy and taxation problems: an update. Working Paper No. 578. Rochester Center for Economic Research, University of Rochester

Thomson W, Yeh C (2008) Operators for the adjudication of conflicting claims. J Econ Theory 143:177-198. doi:10.1016/j.jet.2007.12.005

Tobin J, Pechman J, Mieszkowski P (1967) Is a negative income tax practical? Yale Law J 77:1-27

Young P (1987) On dividing an amount according to individual claims or liabilities. Math Oper Res 12: $198-414$ 